\title{
A Conceptual Design Study for Active Nondestructive Assay System by Photon Interrogation for Uranium-Bearing Waste with MVP Code and Evaluated Photonuclear Data
}

\author{
Takeshi SAKURAI ${ }^{1, *}$, Kazuaki KOSAKO ${ }^{2}$ and Takamasa MORI ${ }^{1}$ \\ ${ }^{1}$ Japan Atomic Energy Agency, 2-4 Shirakata, Tokai-mura, Naka-gun, Ibaraki-ken, 319-1195, Japan \\ ${ }^{2}$ Shimizu Corporation, 3-4-17 Etchujima, Koto-ku, Tokyo, 135-8530, Japan
}

\begin{abstract}
A conceptual design study has been carried out with a Monte Carlo transport code on an active nondestructive assay system for low-level uranium-bearing wastes discharged from an uranium enrichment plant. Delayed neutrons from photofissions of uranium isotopes in the steel waste are counted with this system to confirm that the activity concentration of uranium in this waste is below the clearance level. The present design work needs a coupled neutron/photon transport code which can simulate the photonuclear reaction and the resultant emission of neutrons. We have modified a continuous-energy Monte Carlo transport code MVP to equip it with a function to simulate the emission of photoneutrons along with the delayed neutrons from the photofissions. The photonuclear data library for the modified MVP code was prepared on the basis of evaluated photonuclear data by IAEA. For the ${ }^{235} \mathrm{U}$ and ${ }^{238} \mathrm{U}$, we employed evaluated data in the JENDL photonuclear data 2004, which stores complete delayed neutron emission data for these nuclides. With this code system, a time-dependent simulation of neutron counts was made for the assay system, which consisted of a $15 \mathrm{MeV}$ end-point bremsstrahlung photon source, helium-3 neutron detectors and the waste. The present paper describes the modification to the MVP code and the results of design study.
\end{abstract}

KEYWORDS: photon interrogation, photofission, delayed neutron, MVP code, photonuclear data, uraniumbearing waste, clearance level

\section{Introduction}

Various kinds of low-level radioactive wastes of metal arise in the nuclear fuel cycle. Recycling and reuse of them, after their activities being found to be under the "clearance level"1) through assay, is an important issue to reduce the amount of radioactive wastes disposed of and to further make the wastes to be a valuable resource. For example, a significant amount of low-level steel wastes will be discharged from an uranium enrichment plant, through replacement of cylinders for gas centrifuge process. It is therefore necessary to develop an assay technology to confirm that the activities in these "uraniumbearing wastes" are under the clearance level.

Active nondestructive assay(NDA) techniques have been developed for applications such as inspecting nuclear materials for the safeguards and assaying activity of transuranics in bulk solid wastes. ${ }^{2,3)}$ The major uranium isotopes have much longer half lives than the other actinides such as plutonium isotopes. This feature of uranium isotopes often makes quantitative determination of them in the waste to be difficult by means of detecting characteristic radiations spontaneously emitted from them with the nondestructive manner. This leads the active NDA technique to be more efficient than a passive one, for the uranium-bearing wastes in many cases.

In the present study, we applied the active NDA technique

*Corresponding author, E-mail:sakurai.takeshi@jaea.go.jp by photon interrogation to assaying the activity concentration in the low-level uranium-bearing wastes and carried out a conceptual design for an assay system with this technique. The waste package is irradiated with high energy photons produced by a pulsed electron accelerator to induce photofissions of uranium in the waste. The resultant fission products emit a small number of delayed neutrons which are counted by detectors placed near the waste during the period between the photon flashes(electron pulses) to measure the activity of uranium.

For a numerical simulation in this design work, a continuous-energy neutron/photon Monte Carlo transport code $\mathrm{MVP}^{4}$ ) of Japan Atomic Energy Agency(JAEA) was modified to be equipped with a function to calculate the emission of the photoneutrons along with the delayed neutrons from the photofission. Similar numerical simulations on the active NDA have been addressed by several authors in recent years by the use of the MCNP and/or MCNPX with the photonuclear physics, ${ }^{5,6)}$ but most of them have been focused on a methodology to detect a nuclear material concealed in a cargo, for the homeland security. ${ }^{7,8)}$ In the present study, an active NDA system by the photon interrogation is proposed for the assay of low-level wastes which contain far less amount of uranium than the above-mentioned applications for the homeland security. The steel waste discharged from the uranium enrichment plant is the target of present study. The goal of 
study is to draw a requirement for the feasibility of proposed system to confirm that the activity of this waste is below the clearance level. For this goal, the result of simulation with the modified MVP is used to evaluate the lower limit of detection for the activity concentration in the target waste by the proposed system.

The photonuclear interaction is a rare event due to its small cross-section when it is compared to the competing photoatomic one. Furthermore, the target wastes in the present study contain only a small amount of uranium as mentioned above. A new variance reduction technique is therefore also introduced to the modified MVP for an efficient simulation of proposed active NDA system.

Section II of the present paper shows the photonuclear data used for the present study. The modifications to the MVP is described in Section III. Results of numerical simulation for the proposed active NDA system are presented in Section IV. The lower limit of detection for the activity concentration by the proposed system is also evaluated with the use of these results in this section.

\section{Photonuclear Data for Modified MVP}

The photonuclear reaction is caused by incident photons of energies higher than the reaction thresholds. As the result, nuclear particles are emitted with emission spectra similar to the neutron-induced reactions. Photonuclear data evaluations for more than 160 nuclides, including cross sections and such emission spectra of secondary particles, were performed under the Co-ordinated Research Project on "Compilation and Evaluation of Photonuclear Data for Applications"9) by the International Atomic Energy Agency. This IAEA library was used in the present work. For the major uranium isotopes of ${ }^{235} \mathrm{U}$ and ${ }^{238} \mathrm{U}$, we employed parts of evaluated data in the JENDL photonuclear data 2004(JENDL/PD-2004), ${ }^{10)}$ which has stored complete delayed neutron emission data including a yield along with relative abundances, decay constants and energy spectra for six precursor groups, due to the photofission of these nuclides.

The photofission cross sections of these uranium isotopes are compared between the IAEA library and JENDL/PD-2004 in Appendix A. The active NDA system is designed in the present study for the low enriched uranium as will be described in Section IV. 1. The principal source of delayed neutrons therefore is the ${ }^{238} \mathrm{U}$. The photofission cross-section of this nuclide in the JENDL/PD-2004 is similar to that in the IAEA library in the energy range below $15 \mathrm{MeV}$ which is important for the present active NDA system as will also be mentioned in Section IV. 1.

These evaluated data libraries have covered most of the nuclides needed for the simulation of present active NDA system, and have been given for incident photon energies from the photonulear reaction threshold to the upper limit ranging between 20 and $150 \mathrm{MeV}$. These evaluated data therefore are suitable for the numerical simulation in the present study.

The ENDF-6 format ${ }^{11)}$ has been adopted in these evaluations, so we processed them to generate the photonuclear data library for the modified MVP, by the use of a code system developed for processing the evaluated nuclear data file of neutron-induced reactions in this format ${ }^{12)}$ after making several modifications to the system. This code system is capable of processing data for most of reaction channels given in the evaluated photonuclear data files. Some of these data files have not explicitly given the yields of secondary particles. In such cases, production cross sections for these particles have been evaluated and were used to calculate the yields to be stored in the library for the modified MVP.

The photonuclear data evaluations usually include the data for reactions which emit the charged particles such as proton. The photonuclear data for these particles however were not used in the modified MVP as this code has been made for the transport calculation of the neutron and photon.

\section{Modifications to MVP}

\section{Photoneutron Production}

The modified MVP adopts the total photon interaction cross-section calculated by summing the photoatomic and photonuclear ones. The total cross-section is then used to sample the distance to the next photon collision. The contribution of photonuclear reaction is at most a few percent of the total interaction, and hence the addition of photonuclear cross-section usually induces a negligibly small impact on this sampling.

The photonuclear reaction, by contrast, is the primary source of neutron which is essential for the active NDA technique by the photon interrogation of present study. The modified MVP is focused on describing behavior of the photoneutrons including the delayed neutrons due to the photofission. Their emission is simulated at the photon collision site with a following procedure.

As a first step, a nuclide collided with is sampled. The collision type for the chosen nuclide is then sampled between the photonuclear and photoatomic. If the collision is the photoatomic, a routine for this collision type, which has already been available in the original MVP, is used. If the photonuclear collision is chosen, a newly installed routine further samples a type of photonuclear reaction. As a final step, an integer number of the emitted photoneutrons and/or photons, their energies and directions are sampled, by the use of the yield, energy and angular distributions given for the chosen reaction type in the library.

When the reaction type is determined to be the photofission, both prompt and delayed neutrons are generated. The relative abundances are then used for sampling one of the precursor groups to which each of the generated delayed neutrons belongs. A period from the photofission to the delayed neutron emission is also probabilitically determined using the decay constant of this sampled group.

\section{Enhanced Tally}

The original MVP's tallies of particle flux and reaction rates for a particular spatial region, energy group, time bin, nuclide and type of reaction are also available in the modified MVP where the photonuclear reaction rate can also be tallied. The tally has been enhanced in the modified MVP, where the flux and reaction rates are tallied for the photoneutrons that are generated from a particular origin. The followings are avail- 
able as such origins: (a) the region where the photoneutron was born, (b) the nuclide that emitted the photoneutron and (c) the reaction type that generated the photoneutron. The prompt and delayed neutrons generated by the photofission are treated as they have the different origins of reaction type to separate the tallies between them. To make this enhancement, each of the photoneutrons holds the information regarding its origins listed above in the modified MVP.

When the photoneutron tracking is terminated with a reaction that emit secondary neutrons, such as the fission and inelastic scattering, these secondary neutrons also have their primary origins in the photonuclear reaction. In such cases, the information concerning the photoneutron origins is transferred to these secondary neutrons, so that their contributions are added to the foregoing tallies of photoneutrons.

When the uranium-bearing waste is irradiated with the high energy photons, a material that composes a waste matrix emits a considerable amount of photoneutrons which could contribute the neutron detector counts of active NDA system and could work as an obstacle to assaying the activity of uranium in the waste. The present enhancement of tally aims to evaluate an influence of these photoneutrons. While this enhanced tally is similar to the tally tagging in the MCNPX, ${ }^{13)}$ the enhancement is optimized for the numerical simulation in the present design study.

\section{Biasing Techniques for Variance Reduction in Tally of Photoneutrons}

Some of the foregoing calculation steps are coded in the modified MVP with biasing techniques that are aimed at the variance reduction in the photoneutron tallies for the efficient simulation of active NDA system for the low-level uraniumbearing waste. Among these techniques, the principal ones are described briefly as follows.

First, atomic number densities of uranium isotopes in the target low-level waste are far less than those of ion isotopes which compose the matrix of waste by a factor of $1 \times 10^{-3}$ or less, while the total photon interaction cross-section of uranium is larger than that of iron by a factor of 10 below $15 \mathrm{MeV}$ . The photons therefore rarely collide with the uranium isotopes when the analog Monte Carlo method is adopted because the probability $P_{i}$ that the photon collides with a particular nuclide $i$ (e.g. one of uranium isotopes) is calculated in this method by

$$
P_{i}^{\text {analog }}=\frac{\rho_{i} \sigma_{i}}{\sum \rho_{k} \sigma_{k}}
$$

where $\rho_{k}$ and $\sigma_{k}$ are the atomic number density of the nuclide $k$, and its total photon interaction cross-section, respectively. The summation in the denominator is for all the nuclides in a region including the collision site. This feature could cause poor statistics in the tallies of photoneutrons emitted from the uranium isotopes, which would lead to a difficulty in the simulation of present study.

To overcome it, we introduced a new biasing technique to sample more often the uranium as the nuclide collided with. The biased sampling makes the photon to collide with nuclides in a group $G$ (e.g. uranium isotopes) with a larger probability $P F C N(<1)$ specified by an user of code, so that the biased collision probability for a nuclide $i$ in $G$ is given by

$$
P_{i}^{\text {bias }}=P F C N \frac{\rho_{i} \sigma_{i}}{\sum_{m \in G} \rho_{m} \sigma_{m}} .
$$

That for a nuclide $j$ not being a member of $G$ is given by

$$
P_{j}^{\text {bias }}=(1-P F C N) \frac{\rho_{j} \sigma_{j}}{\sum_{n \notin G} \rho_{n} \sigma_{n}} .
$$

With this biasing, the weight of incident photon is adjusted and given by

$$
W_{i}^{\mathrm{bias}}=W_{0} \frac{\sum_{m \in G} \rho_{m} \sigma_{m}}{\sum \rho_{k} \sigma_{k}} \frac{1}{P F C N},
$$

and

$$
W_{j}^{\text {bias }}=W_{0} \frac{\sum_{n \notin G} \rho_{n} \sigma_{n}}{\sum \rho_{k} \sigma_{k}} \frac{1}{(1-P F C N)},
$$

for those that collide with the nuclide $i$ and $j$, respectively, where the $W_{0}$ is the weight of photon before this sampling. The region-dependent probability for this biased sampling is available in the modified code.

Secondly, the photonuclear interaction is a rare event due to its small cross-section when it is compared to the photoatomic one as mentioned above. A biasing technique to sample more often the photonuclear event is therefore required for the variance reduction in the tally of photoneutrons. ${ }^{5,6)}$ The modified code has a capability to sample the photonuclear event with a probability $P P N B R(<1)$ chosen by the user of code for each nuclide included in the problem to be solved, where this probability can be larger than that derived from a ratio of photonuclear to photoatomic cross sections. With this biasing, the weight of incident photon is adjusted and given by

$$
W_{p n}^{\mathrm{bias}}=W_{0}^{\prime} \frac{\sigma_{p n}}{\sigma_{p n}+\sigma_{p a}} \frac{1}{P P N B R},
$$

and

$$
W_{p a}^{\mathrm{bias}}=W_{0}^{\prime} \frac{\sigma_{p a}}{\sigma_{p n}+\sigma_{p a}} \frac{1}{1-P P N B R},
$$

for those that undergo photonuclear and photoatomic event, respectively, where $W_{0}^{\prime}, \sigma_{p n}$ and $\sigma_{p a}$ are the weight of incident photon before this sampling, the photonuclear reaction cross-section of the nuclide collided with and the photoatomic interaction one, respectively. When this biasing technique is applied with that for sampling the nuclide collided with, the photon weight $W_{i}^{\text {bias }}$ or $W_{j}^{\text {bias }}$ is employed as the $W_{0}^{\prime}$. The two biasing techniques were validated through confirming an equivalent tally result between the biased and unbiased calculations.

Although these biases in sampling could induce a significantly large variation in the incident photon weight after the sampling, the photoneutrons can be generated with a constant weight chosen by the user. The integer number of photoneutrons is probabilitically determined to preserve the expected value $\left\langle N_{i m t}\right\rangle$ for each nuclide $i$ sampled to be collided with and for each photonuclear reaction $m t$ sampled to occur :

$$
\left\langle N_{i m t}\right\rangle=\frac{Y_{i m t} W_{i p n}^{\text {bias }}}{W G T P N_{i m t}}
$$




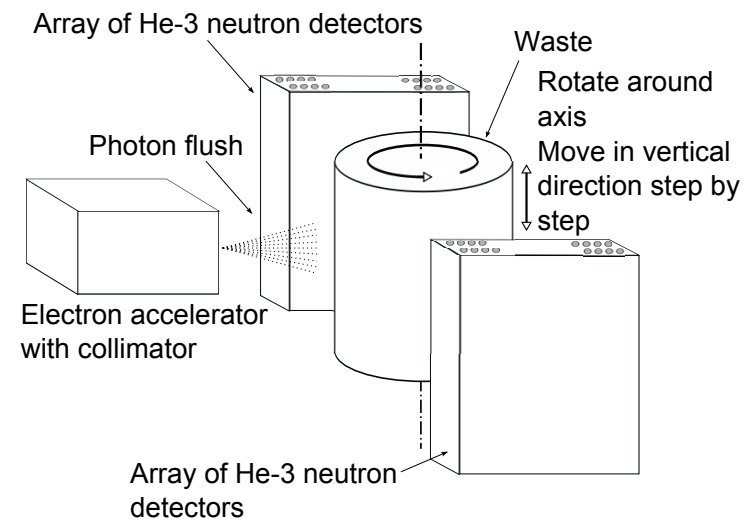

Fig. 1 Schematic view of proposed active NDA system.

where $Y_{i m t}, W_{i p n}^{\text {bias }}$ and $W G T P N_{i m t}$ are the photoneutron yield of reaction $m t$ of nuclide $i$, the adjusted weight of incident photon after the foregoing biased samplings and the weight of generated photoneutron that is specified by the user for the nuclide $i$ and reaction $m t$. A region-dependent value can also be chosen for the weight $W G T P N_{i m t}$. The treatment mentioned here is obtained by applying a procedure for fission neutron production in the original $\mathrm{MVP}^{4)}$ to that for the photoneutron production. The photon production by the photonuclear reaction is treated in the same manner.

\section{Numerical Simulation for Active NDA System}

\section{Overview of Proposed System}

It is a trend throughout the world that the steel wastes are melted and then re-casted before being recycled. ${ }^{14)}$ Figure 1 depicts a schematic view of the proposed active NDA system which is composed of the electron accelerator with a photon collimator and two arrays of ${ }^{3} \mathrm{He}$ neutron detectors for assaying the re-casted steel waste of a cylindrical shape. This system is housed in an assay room of $8 \mathrm{~m}$ length, $4 \mathrm{~m}$ width and $4 \mathrm{~m}$ height.

The electron energy chosen was $15 \mathrm{MeV}$ for the following reason. The photon with energy higher than $15.9 \mathrm{MeV}$ induces $(\gamma, \mathrm{p})$ reaction of ${ }^{18} \mathrm{O}$ which exists in the air, and the produced ${ }^{17} \mathrm{~N}$ decays to ${ }^{17} \mathrm{O}$ with the half-life of $4.2 \mathrm{~s}$. The ${ }^{17} \mathrm{O}$ then decays to ${ }^{16} \mathrm{O}$, emitting a neutron, with the half-life of $0.04 \mathrm{~s}$. This neutron could be counted during a short period between photon flushes and thus could be contamination to the delayed neutron counts.

The waste is moved during the assay in a manner such that its level is changed step by step as shown in Fig. 1 to make an almost uniform exposure for the whole of waste in the vertical direction. In addition, the waste is rotated around its vertical axis with a constant speed.

The proposed system is applied to assaying various kinds of steel wastes, such as the carbon steel and stainless steel, after they are melted and re-casted. The principal constituent in all of them is the iron, but there are variations in the small amount of alloying elements among them. Furthermore, the re-casting process usually produces some pores in the steel, which decrease the bulk density of steel typically by $20 \%$ after this process. Another variation, hence, could be found in the den-
Table 1 Specific activity of uranium with various ${ }^{235} \mathrm{U}$ enrichment $^{15)}$

\begin{tabular}{lc}
\hline Enrichment $(\%)$ & Activity $(\mathrm{Bq} / \mathrm{g}$ of U) \\
\hline 0.45 (depleted uranium) & $1.8 \times 10^{4}$ \\
0.72 (natural uranium) & $2.6 \times 10^{4}$ \\
1.0 & $2.8 \times 10^{4}$ \\
1.5 & $3.7 \times 10^{4}$ \\
5.0 & $1.0 \times 10^{5}$ \\
\hline
\end{tabular}

sity among the cylinders of re-casted steel. The present assay system is based on counting the delayed neutrons leaking from the waste matrix as mentioned in Section I. These neutrons are generated in the matrix by the uranium photofissions caused by the photons from the accelerator. Some of these neutrons and photons are absorbed principally by the iron when they travel through the matrix made of the re-casted steel. The variations in the bulk density of the steel therefore has an impact on the number of counts, but those in the amount of the alloying materials have a far less influence on this number than the density. The melting and re-casting processes decrease the density of steel as mentioned above, and lead to an increase of the delayed neutron counts. The density of steel before these processes was adopted for the present simulation to assess the feasibility of the present system, because this density gives the less number of counts and the poorer results for the detection limit of the uranium activity concentration in the waste than would be found after the processes.

From these reasons, it was assumed in the simulation that the waste matrix had the density and composition of typical carbon steel and was contaminated with the uranium as will be described below. Furthermore, the matrix potentially includes a small amount of oxygen and fluorine. The origin of the latter is the uranium hexafluoride $\left(\mathrm{UF}_{6}\right)$ used in the enrichment process. They should be taken into account as the background $(\alpha, \mathrm{n})$ neutron source with the uranium isotopes as will be mentioned in Section IV. 4 rather than the photoneutron source. The assumed atomic composition of matrix will be shown in Section IV. 3.

A parametric study was carried out for the size of target waste. The diameter of it was varied as 20,30 and $40 \mathrm{~cm}$, and the height of it was set to be equal to the diameter. The waste matrices with the typical density of $7.85 \mathrm{~g} / \mathrm{cm}^{3}$ covered a wide range in weight from 50 to $395 \mathrm{~kg}$ in the present study.

The waste discharged from the uranium enrichment plant is contaminated by the uranium with various enrichment which is usually not more than $5 \%$. The natural uranium contains a small amount $(0.0053 \%)$ of ${ }^{234} \mathrm{U}$. This minor isotope has the half-life shorter than those of major uranium isotopes by a factor of $1 \times 10^{-4}$ or less and its concentration increases during the enrichment process. The uranium of higher enrichment therefore has the more specific activity as shown in Table $\mathbf{1 .}$

Figure 2 shows a breakdown of the specific activity of uranium which was estimated using the half lives of three uranium isotopes ${ }^{234} \mathrm{U},{ }^{235} \mathrm{U}$ and ${ }^{238} \mathrm{U} .{ }^{16)}$ Among them, the ${ }^{234} \mathrm{U}$ and ${ }^{238} \mathrm{U}$ have dominant contributions and their contributions vary with the enrichment.The isotopic content of ${ }^{234} \mathrm{U}$, how- 


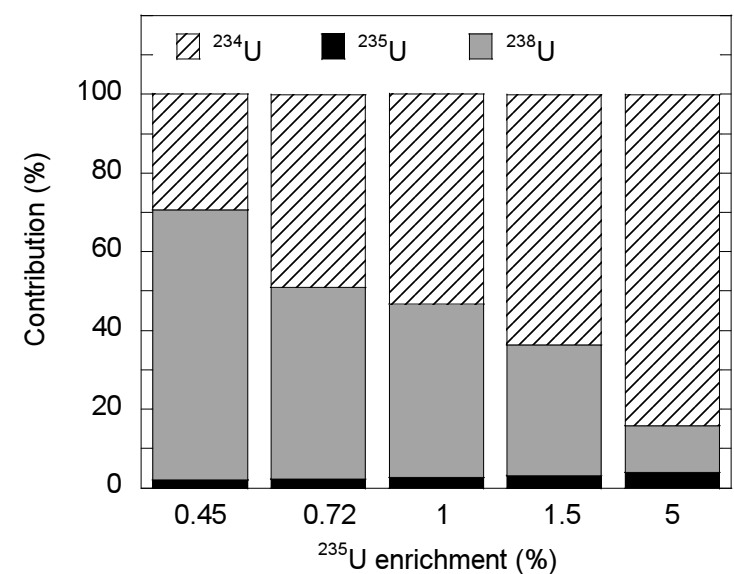

Fig. 2 Breakdown of activity of uranium with various ${ }^{235} \mathrm{U}$ enrichment

ever is estimated to be at most $0.037 \%$ for the uranium with enrichment not more than 5\%, and hence the ${ }^{238} \mathrm{U}$ and ${ }^{235} \mathrm{U}$ have dominant contributions to the photofissions. It should be pointed out that the nuclides that determine the activity differ from those that dominate the photofissions in some cases.

From these features of the waste to be assayed, the delayed neutron counts of major uranium isotopes, the ${ }^{238} \mathrm{U}$ and ${ }^{235} \mathrm{U}$, are used by the present system. Furthermore, the waste is assumed to be contaminated by the $5 \%$ enriched uranium which has the maximum specific activity as shown in Table 1 to confirm that the activity concentration of waste does not exceed the clearance level.

The target waste was once melted and then re-casted before being assayed. Most of the wastes therefore have almost uniform contamination of uranium, but the others have the distributed one. The latter waste is in general more difficult to assay than the former one. In the present design work, we carried out the simulation for a hypothetical case of localized contamination. This case brings the most difficult assay because a difference in the detector counts of delayed neutrons could arise between the different radial positions of the contaminated region and this difference should be taken into account in the assay.

\section{Specification of Proposed System}

A typical operation condition of electron accelerator was used in the present simulation. The accelerator is operated in the pulsed mode at a frequency of $200 \mathrm{~Hz}$. A beam current at a pulse peak and a pulse width are $142 \mathrm{~mA}$ and $4.5 \mu \mathrm{s}$, respectively, which correspond to an average beam current of $128 \mu \mathrm{A}$. A gold converter of $0.9 \mathrm{~mm}$ thickness and $4 \mathrm{~mm}$ diameter with a copper backing of $2.5 \mathrm{~mm}$ thickness is used to generate the photons, part of which then passes through a copper collimator. The number of incident electrons on the converter amounts to $4.0 \times 10^{12}$ per pulse.

Figure 3 shows an arrangement of the high energy photon source, the collimator, the waste and the detector arrays. The collimator is open for the photons of direction within typically 5 degrees angle around the direction of electron beam. This angle was chosen to keep the detector arrays away from a large

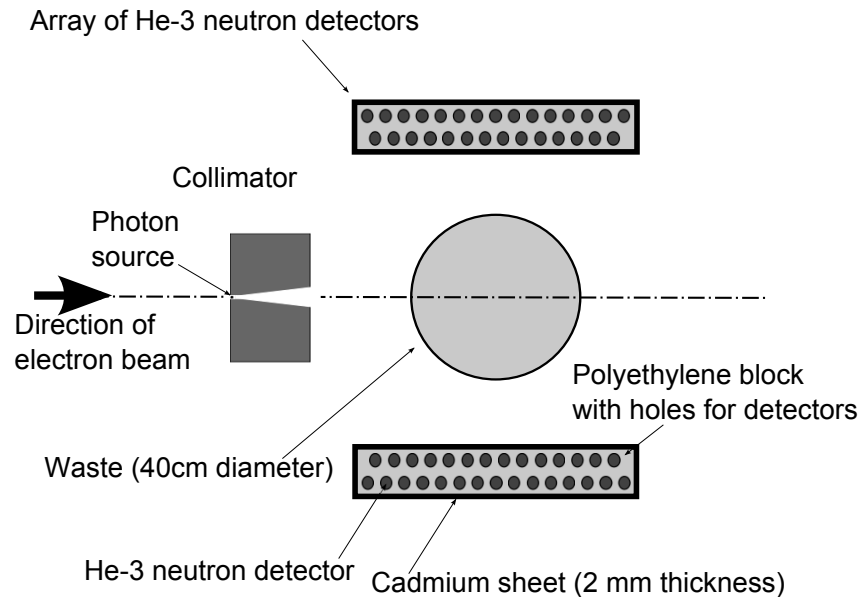

Fig. 3 Horizontal cross-sectional view at the photon source elevation for the calculation model of proposed active NDA system.

exposure. The distance between the photon source and the center of waste is $60 \mathrm{~cm}$.

A difference of $3 \mathrm{~cm}$ between the levels was chosen to move the waste for the uniform exposure in the vertical direction, where an angular distribution of collimated photons was taken into consideration. The different levels of 9, 13 and 17 steps are used to cover the whole waste of 20, 30 and $40 \mathrm{~cm}$ height, respectively. The waste is rotated with a constant speed of 360 degrees for each $3 \mathrm{~min}$. The number of electron pulses during $3 \mathrm{~min}$ amounts to 36,000 . At each level, the delayed neutron counts due to the photofission of uranium are acquired in a period between these pulses as will be described in Section IV. 4. A total assay time required for one waste is between $51 \mathrm{~min}$ and $1 \mathrm{~h} 40 \mathrm{~min}$.

In addition, background counts are measured for $10 \mathrm{~min}$ when the accelerator is not operated. This background counting time was chosen in a manner such that it was not more than the assay time for the one waste mentioned above and thus did not make any considerable influence on the process of assaying a lot of wastes.

Each of the detector arrays consists of $29{ }^{3} \mathrm{He}$ tubes of $2.5 \mathrm{~cm}$ outer diameter and $100 \mathrm{~cm}$ active length with 4 atmospheres filled pressure. The detectors are put in holes of polyethylene blocks $13 \mathrm{~cm}$ width $\times 55 \mathrm{~cm}$ length $\times 159 \mathrm{~cm}$ height as a neutron moderator. A distance between the centers of two adjacent holes is $3.4 \mathrm{~cm}$ in the direction along the electron beam and $6.7 \mathrm{~cm}$ in the other direction. The entire assembly of detector array is then wrapped with a cadmium sheet of $2 \mathrm{~mm}$ thickness. The distance between the two assemblies is $67 \mathrm{~cm}$.

\section{Calculational Methodology}

The present numerical simulation involved a two step approach: (1) a time-independent electron-photon transport calculation to obtain energy and angular distributions of bremsstrahlung photons leaking from the converter, and (2) a time-dependent photon-neutron transport calculation to obtain the ${ }^{3} \mathrm{He}$ detector counts of delayed neutrons. 
The calculation of step (1) was carried out for a model of converter with an incident electron source of $15 \mathrm{MeV}$ on the surface of it, using the MCNP4C code. The photons produced by bremsstrahlung in the converter and leaking from it were tallied, which were then used as the energy and angular distributions of leaking photons. This distribution was calculated with a mesh width of 1 degree for the polar angle around the direction of electron beam injection, with an energy mesh width of $0.3 \mathrm{MeV}$. These distributions will be used for the source of calculation step (2). The energy cut off was set at $2.1 \mathrm{MeV}$ which corresponded to the lowest photonuclear threshold energy of nuclides included in materials in a model of calculation step (2). A number of all the leaking photons with the energy above $2.1 \mathrm{MeV}$ was found to be $0.5478 \pm$ $0.03 \%(1 \sigma)$ per source electron.

The calculation step (2) was carried out by the use of the modified MVP code with a model composed of a point photon source, the collimator, the waste, the assemblies of detector array as shown in Fig. 3. An equipment to move and rotate the waste which was placed under the waste and the assay room with concrete walls that housed the entire system were also modeled. The neutron counts at the ${ }^{3} \mathrm{He}$ detectors were simulated by a tally of ${ }^{3} \mathrm{He}(\mathrm{n}, \mathrm{p})^{3} \mathrm{H}$ reactions in the active regions of detectors.

The foregoing localized contamination of uranium was simulated by placing a small contaminated region of a spherical shape in the waste. The diameter of sphere chosen was $3 \mathrm{~cm}$ for the waste of $40 \mathrm{~cm}$ diameter and $2 \mathrm{~cm}$ for those of the smaller sizes. Three cases were considered for the radial position: (a) the center, (b) several positions near the half of radius and (c) the outside edge, to obtain the difference in the detector counts of delayed neutrons between the different radial positions.

For the cases (b) and (c) where the contaminated region is not placed at the center in the radial direction, the region turns in a circle around the vertical axis of waste with a constant speed as the waste rotates during the assay. In these cases, the contaminated region was modeled in the calculation by the use of a torus that corresponded to the path of turning sphere region. In the vertical direction, the contaminated region was placed at the center of waste to yield a typical result of simulation, as the almost uniform exposure was made for the whole of waste in this direction as mentioned above.

Table 2 lists the atomic number densities used in the model for the contaminated region of case (a) in the waste of $40 \mathrm{~cm}$ diameter for instance. The densities except for the uranium were calculated from a typical composition of carbon steel with $7.85 \mathrm{~g} / \mathrm{cm}^{3}$ density, and were also used for the uncontaminated region in the matrix. One of the isotopes of uranium, ${ }^{234} \mathrm{U}$, was not modeled but its density was added to that of ${ }^{238} \mathrm{U}$, as the ${ }^{234} \mathrm{U}$ had a small contribution to the photoneutron production because of its small content in the uranium. Furthermore, the oxygen and fluorine were not modeled as their densities were difficult to estimate but were expected to be so small that their contributions to the photoneutron production were negligibly small. These uranium densities were chosen in a manner such that "an average activity concentration", defined as a ratio between the total activity of uranium included
Table 2 Atomic number densities of contaminated region (a) in the waste of $40 \mathrm{~cm}$ diameter used for calculation model

\begin{tabular}{cccc} 
& & & $\left(\right.$ atoms $/ \mathrm{cm}^{3}$ ) \\
\hline Nuclide & Density & Nuclide & Density \\
\hline${ }^{12} \mathrm{C}$ & $1.168 \times 10^{21}$ & ${ }^{55} \mathrm{Mn}$ & $1.377 \times 10^{21}$ \\
${ }^{13} \mathrm{C}$ & $1.299 \times 10^{19}$ & ${ }^{54} \mathrm{Fe}$ & $4.813 \times 10^{21}$ \\
${ }^{31} \mathrm{P}$ & $6.105 \times 10^{19}$ & ${ }^{56} \mathrm{Fe}$ & $7.611 \times 10^{22}$ \\
${ }^{32} \mathrm{~S}$ & $5.603 \times 10^{19}$ & ${ }^{57} \mathrm{Fe}$ & $1.825 \times 10^{21}$ \\
${ }^{33} \mathrm{~S}$ & $4.423 \times 10^{17}$ & ${ }^{58} \mathrm{Fe}$ & $2.323 \times 10^{20}$ \\
${ }^{34} \mathrm{~S}$ & $2.483 \times 10^{18}$ & ${ }^{235} \mathrm{U}$ & $3.575 \times 10^{18}$ \\
${ }^{36} \mathrm{~S}$ & $1.179 \times 10^{16}$ & ${ }^{238} \mathrm{U}$ & $6.707 \times 10^{19}$ \\
\hline
\end{tabular}

in the contaminated region and the mass of the whole waste, equalled to the clearance level of $0.1 \mathrm{~Bq} / \mathrm{g} .{ }^{1)}$ For the wastes of smaller sizes and for each of the torus regions, the atomic number densities of uranium isotopes were chosen in the same manner as described above.

Although the torus model thus corresponds to the pass of turning sphere region that is not placed at the center in the radial direction, the volume of torus is larger than that of sphere by a factor of at most 66 in the wastes considered in the present design study. Accordingly, the density of uranium is diluted by this factor in the torus to keep the average activity concentration of the whole waste to be $0.1 \mathrm{~Bq} / \mathrm{g}$ of waste. A series of sensitivity calculation then was carried out as shown in Appendix B to estimate an uncertainty caused by this uranium density dilution in the torus model. As a result, a systematic uncertainty of about $1 \%$ was estimated to be introduced by adopting this model.

In Table 2, the atomic number density of ${ }^{238} \mathrm{U}$ is found to be less than that of ${ }^{56} \mathrm{Fe}$ by a factor of about $1 \times 10^{-3}$. This is a typical example to show that the atomic number densities of uranium isotopes in the target waste are far less than that of principal nuclide ${ }^{56} \mathrm{Fe}$ which is included in the matrix of target waste. The biasing techniques described in Section III. 3 were therefore required. The collision probability $P F C N$ of 0.9 was used in the biased sampling for the uranium isotopes when the photoneutrons from these isotopes were important. We further used the sampling probability $P P N B R$ of 0.9 to sample the photonuclear event more often than the photoatomic one.

\section{Results and Discussions}

(1) Results of Simulation for Delayed Neutron Counts

Figure 4 shows a time-dependent behavior of counts at the detectors due to the photoneutrons promptly emitted at the photon flush. Those originated from all the materials in the simulation model were tallied with successive time bins of $10 \mu \mathrm{s}$ width. When these photoneutrons induced a reaction of uranium isotopes and neutrons were emitted by this reaction, contribution from these neutrons were also included in the tally. These photoneutron counts were further broken down into contributions from different origins by the use of the enhanced tally described in Section III. 2. The break down revealed that those originated in photonuclear reaction of ${ }^{56} \mathrm{Fe}$ had a dominant contribution to the counts. It is observed that 


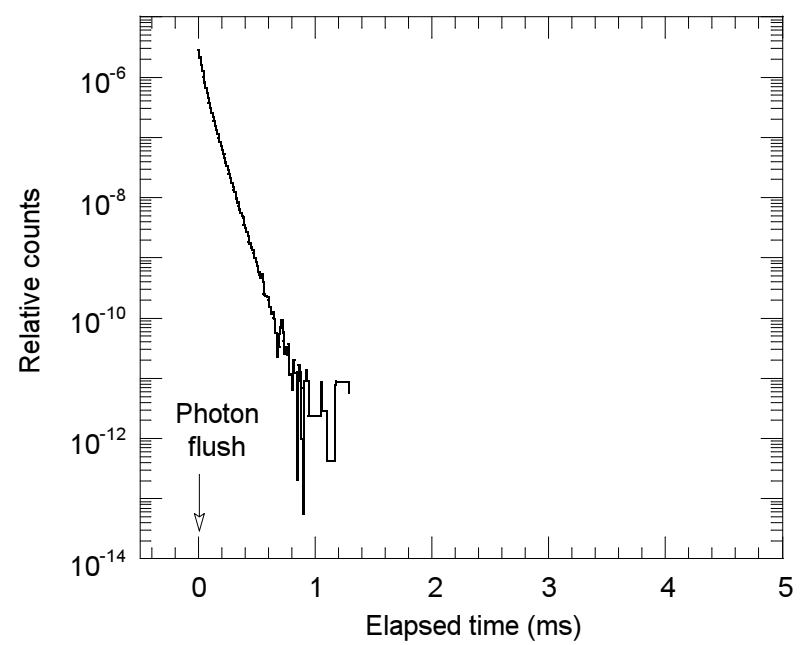

Fig. 4 Time-dependent counts of photoneutrons promptly emitted at the photon flush.

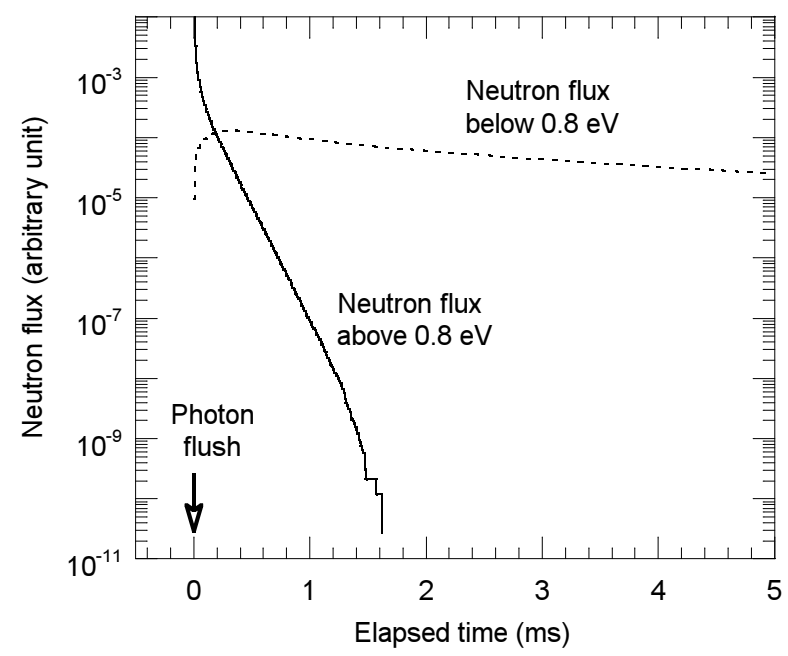

Fig. 5 Time-dependent neutron flux due to the promptly emitted photoneutrons in the assay room.

the number of counts decreases as time elapses after the photon flush and disappears before $2 \mathrm{~ms}$.

This behavior of detector counts was analyzed by the use of the photoneutron fluxes integrated in the vacant spaces in the assay room. Figure 5 shows the time-dependent fluxes of two energy groups with an energy boundary of $0.8 \mathrm{eV}$. The flux above this energy decreases and disappears in the same manner as the detector counts, although the flux below this energy remains in the room after $2 \mathrm{~ms}$. The energy of $0.8 \mathrm{eV}$ corresponds to the cut off energy of $2 \mathrm{~mm}$ thick cadmium which is used to wrap up the detector array. The flux above this energy consequently can contribute to the detector counts and introduces their time-dependent behavior, while the neutrons below this energy are absorbed by the cadmium sheet before reaching the detector and do not contribute to the counts.

Figure 6 presents a typical view for a time-dependent counts at the detectors due to the delayed neutrons from the photofission of uranium, along with those due to the prompt photoneutrons described above, when the accelerator is oper-

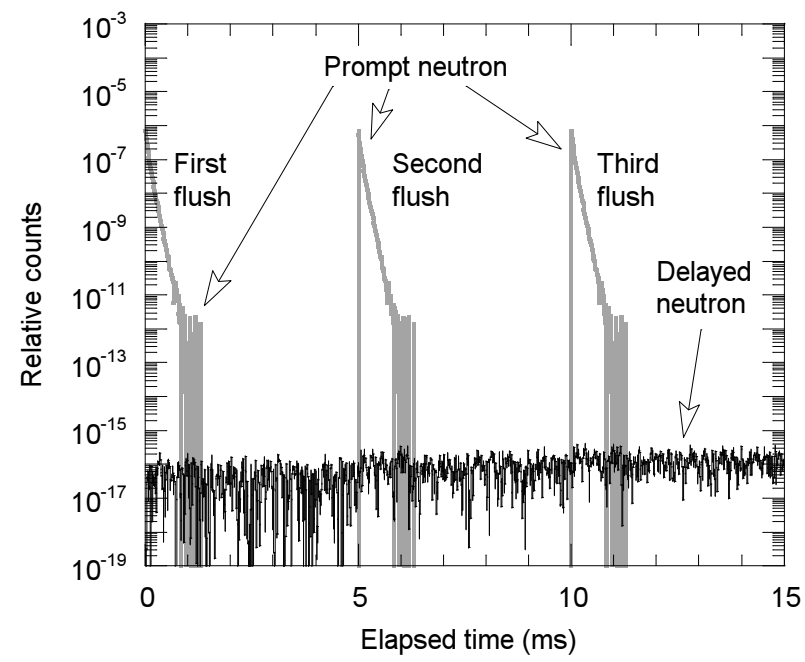

Fig. 6 Time-dependent detector counts of delayed neutrons along with those of prompt neutrons.

ated at $200 \mathrm{~Hz}$. The delayed neutron alone contributes to the detector counts between 2 and $5 \mathrm{~ms}$ after the photon flush. The next flash then comes. The counts in this interval are therefore acquired for each flush to avoid the background counts due to the prompt photoneutrons above. The half lives of most of the delayed neutron precursors are much longer than the short period between the photon flushes as shown in Appendix C. The delayed neutron counts in the interval above hence increase as the number of flushes, as can be observed in Fig. 6, before an equilibrium state is formed. The first delayed-neutronprecursor group has the longest half-life of about $53 \mathrm{~s}$ but this group has the small relative abundance of less than $6 \%$ as tabulated in this appendix. A waiting time of typically 3 min for the equilibrium state is therefore required before the acquisition of counts is started at each of the steps. A total assay time for one waste including this waiting time is $51 \mathrm{~min}, 1 \mathrm{~h} 15 \mathrm{~min}$ and $1 \mathrm{~h} 40 \mathrm{~min}$ for the waste of 20,30 and $40 \mathrm{~cm}$ diameter, respectively.

The total number of delayed neutron counts at each step was calculated from a product of four numbers of (a) the incident electrons per pulse $\left(4.0 \times 10^{12}\right)$, (b) the electron pulses during $3 \mathrm{~min}(36,000)$, (c) the photons generated by bremsstrahlung and leaking from the converter with the energy above $2.1 \mathrm{MeV}$ per source electron $(0.5478 \pm 0.03 \%)$ and (d) the delayed neutron counts per source photon, which were calculated as described above with statistical uncertainties of less than $1.5 \%$ and $0.5 \%(1 \sigma)$ at the center and the other positions, respectively. Furthermore, a loss of counts could take place when the detected neutron pulses are processed through modular electronics instruments. It was assumed that $20 \%$ of detected pulses could not pass the discriminator which was adjusted to reject low-energy noises. Finally, the results of delayed neutron counts of all the steps were summed together to obtain the counts for the whole of one waste with a statistical uncertainty of less than $1.6 \%$ estimated by combining those of (c) and (d) above, in which the latter uncertainty had a dominant contribution.

The numbers of total delayed neutron counts thus calcu- 
lated are plotted in Fig. 7 against the radial distance from the center of waste to the contaminated region. The result practically does not depend on the distance for the waste of $20 \mathrm{~cm}$ diameter, while it depends considerably on the distance for the wastes having larger sizes.

These trend of counts could be brought by following three principal factors which depend on the radial position of contaminated region.

(a) A part of the photons generated at the converter is absorbed by the waste matrix before reaching the contaminated region. The number of photons that arrive at the contaminated region hence decreases as the region approaches the center of waste.

(b) A limited portion of torus in the vicinity of the central axis of collimated photon beam in the side of converter is practically exposed to the photons when the torus is placed close to the outside edge of waste. On the other hand, a large portion of torus is exposed when the torus is placed near the center of waste, because the radius of torus is small and the large portion of it is near the central axis and furthermore the portion of torus in the opposite side to the converter approaches the center of waste and also the converter.

(c) A part of the delayed neutrons produced in the contaminated region is absorbed by the waste matrix before reaching the detector. The number of neutrons that arrive at the detector hence decreases as the region approaches the center of waste.

For the factor (b), the large volume exposed to the collimated photon beam in the torus model corresponds to the long exposure time for the turning sphere region in the actual assay.

When the contaminated region approaches the center of waste, both factors of (a) and (c) work to decrease the counts, while the factor (b) works to increase the counts. For the waste of large size, compensation of the three factors could result in forming the trend of counts considerably dependent on the radial distance as shown in Fig. 7. For the waste of small size, the three factors could work in a moderate manner, and the trend approaches almost flat for the waste of $20 \mathrm{~cm}$ diameter. Further investigations are necessary to confirm these analyses of the trends.

The minimum number of counts appears at the position near the half of radius as shown in this figure. It should be adopted to assess the feasibility of present active NDA system for confirming that the average activity concentration of uranium in the waste is below the clearance level, and was thus used to yield a relation between the average activity concentration and the number of counts as shown in Fig. 8, where it was assumed that the count linearly decreased with the concentration and disappeared at $0 \mathrm{~Bq} / \mathrm{g}$. This relation was then used to estimate the lower detection limit of the average activity concentration as follows.

\section{(2) Estimation of Lower Detection Limit}

The number of counts that corresponds to the lower detection limit is usually estimated from fluctuations in the background counts, because the background counts are subtracted from the gross counts, and the net counts, which correspond to the delayed neutron counts in the present study, could be

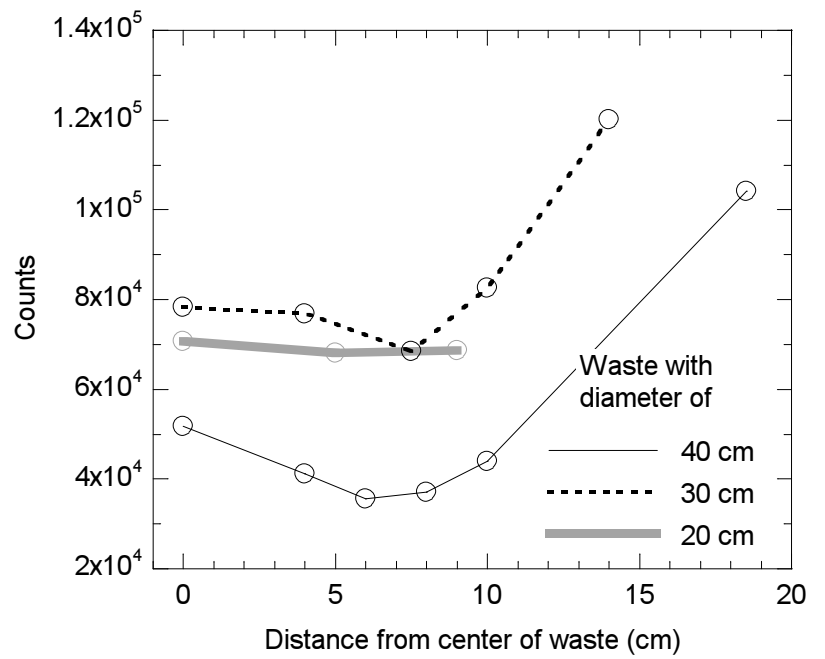

Fig. 7 Total delayed neutron counts dependent on the radial position of contaminated region. The statistical uncertainties of Monte Carlo calculation are less than $1.6 \%$ and $0.6 \%(1 \sigma)$ at the center and the other positions, respectively. They are less than the size of circles.

insignificant if they were not more than the fluctuations in the subtracted background counts. The background is therefore one of the most important parameters to estimate the lower detection limit.

In the present active NDA system, there is no background due to the photoneutrons emitted from the materials except the uranium as described above. The source of background therefore is limited to the neutrons emitted in a natural process such as a reaction caused by high energy cosmic radiations. Furthermore, it is known that the uranium isotopes emit the spontaneous fission neutrons and the $\alpha$-particles emitted by their decay cause $(\alpha, n)$ reaction with light nuclei in the waste. These neutrons also contribute to the background counts. Their contributions however are found to be negligibly small as shown in Appendix D at the lower detection limit which will be discussed below.

The fluctuations in the counts were estimated as the statistical deviation at $3 \sigma$ level in the background counts. No simulation method has been available to estimate the background counts due to the high energy cosmic radiations as mentioned above for the present system. The amount of background counts depends on several conditions such as a location of assay room and can be known from the measurement. A parametric study therefore was carried out by considering several levels of background count rate between 10 and 10,000 cps.

For example, the background counts of $60,000 \pm 1.3 \%$ ( $3 \sigma$ ) are obtained for the 10 min counting when the count rate is $100 \mathrm{cps}$. The waste of $40 \mathrm{~cm}$ height, for instance, needs the total time of $1,836 \mathrm{~s}$ for the delayed neutron counts acquisition for all the 17 steps of different levels. In this case, the background counts that should be subtracted from the gross counts are estimated to be $183,600 \pm 1.3 \%(3 \sigma)$, and consequently, the estimated fluctuation in this background counts is found to be 2,387 counts. The lower detection limit of the average 


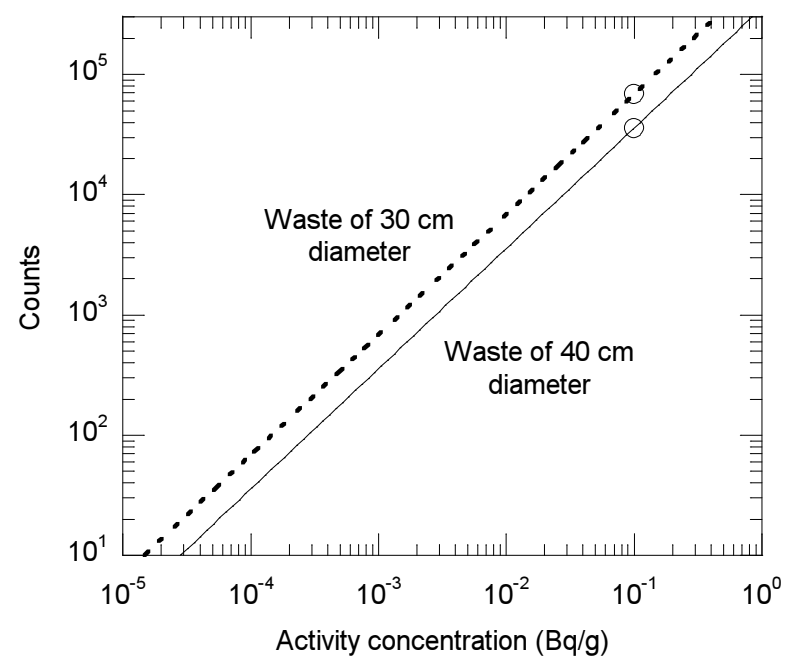

Fig. 8 Relation between the average activity concentration of uranium and the total delayed neutron counts. The statistical uncertainties of Monte Carlo calculation are less than $0.6 \%(1 \sigma)$ at the points of $0.1 \mathrm{Bg} / \mathrm{g}$. They are less than the size of circles. The result for the waste of $20 \mathrm{~cm}$ diameter is not shown as it was practically the same as the result for the waste of $30 \mathrm{~cm}$ diameter.

activity concentration is determined by the use of this fluctuation along with the relation shown in Fig. 8, and is found to be $6.7 \times 10^{-3} \mathrm{~Bq} / \mathrm{g}$ for the waste of $40 \mathrm{~cm}$ diameter.

The fluctuations in the background counts along with the detection limit can be lowered by increasing the time assigned to the background counting. But decreasing the detection limit by a factor of less than 0.1 with this procedure is not realistic in a practical assay, because it is necessary to increase the time for background counting by a factor of more than 100 .

The fluctuations are estimated also for the cases of other background count rates and the waste of smaller sizes. The delayed neutron counts acquisition time for all the 9 and 13 steps are 973 and 1,404 s for the wastes of 20 and $30 \mathrm{~cm}$ diameter, respectively, and were used in this estimation as described in the example above. The results are shown in Fig. 9.

The lower detection limit of less than $0.01 \mathrm{~Bq} / \mathrm{g}$ is in general required for reliability in confirming that the activity concentration of waste is below the clearance level of $0.1 \mathrm{~Bq} / \mathrm{g}$. The delayed neutron counts that correspond to the activity concentration of $0.01 \mathrm{~Bq} / \mathrm{g}$ are obtained from Fig. 8 and shown in Table 3. These counts have the statistical uncertainty of less than $0.6 \%$ in Monte Carlo calculation and the systematic one of $1 \%$ brought by the torus model.

These 'net' counts are then used as the fluctuations in the background counts to give the lower detection limit of $0.01 \mathrm{~Bq} / \mathrm{g}$. Table 3 also presents the background count rates that are drawn from these fluctuations with the relations shown in Fig. 9. The statistical and systematic uncertainties of these count rates are estimated to be less than $1.2 \%$ and $2 \%$, respectively by the error propagation. The proposed system for the detection of activity concentration of clearance level in the target waste is feasible with the background count rates lowered below the values shown in this table. The uncertainties above

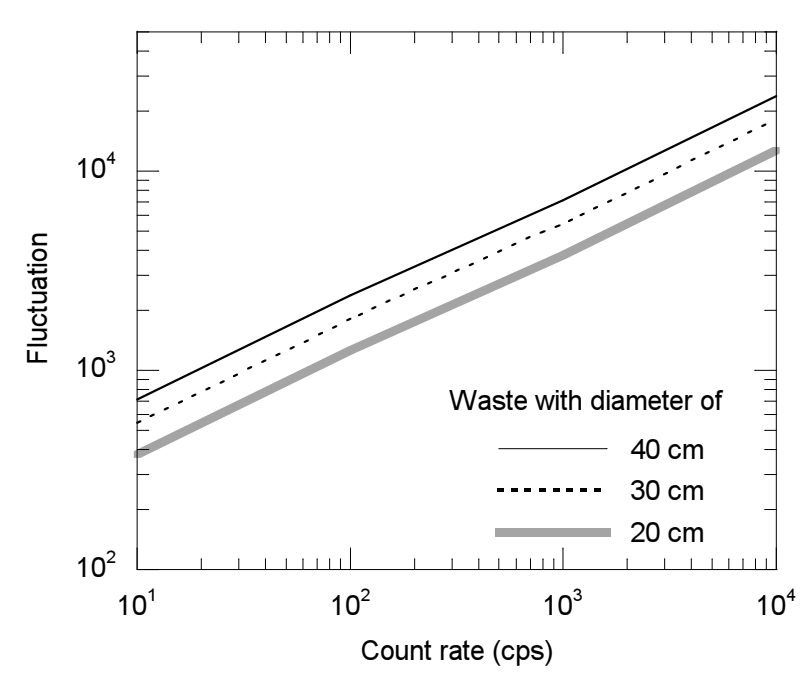

Fig. 9 Fluctuation in background counts against its count rate.

Table 3 Background count rate to achieve lower detection limit of $0.01 \mathrm{~Bq} / \mathrm{g}$

\begin{tabular}{lccc}
\hline Diameter of waste $(\mathrm{cm})$ & 20 & 30 & 40 \\
\hline Fluctuation in background counts $^{a)}$ & 6,800 & 6,850 & 3,560 \\
Background count rate (cps) & 3,260 & 1,580 & 250 \\
\hline
\end{tabular}

a) Delayed neutron counts that correspond to the activity concentration of $0.01 \mathrm{~Bq} / \mathrm{g}$ in Fig. 8

are so small that they do not pose any significant influence on assessing the feasibility of proposed system.

In addition, when the background count rate is found by the measurement to be far less than the above results, the optimization in the design of proposed system, such as a reduction in the accelerator beam current, is possible.

\section{Summary and Conclusion}

The continuous-energy Monte Carlo transport code MVP was modified and equipped with the function to simulate the emission of photoneutrons along with the delayed neutrons due to the photofissions. The photonuclear data library for the modified MVP was prepared for more than 160 nuclides on the basis of evaluated photonuclear data under IAEA Coordinated Research Project. For the photonuclear data of ${ }^{235} \mathrm{U}$ and ${ }^{238} \mathrm{U}$ with their delayed neutron emission data due to the photofission, JENDL/PD-2004 was adopted. The biasing techniques aimed at the variance reduction in the photoneutron tally, along with the enhancement in the tally, were prepared in the modified code for the efficient simulation of active NDA system by the photon interrogation for the assay of low-level uranium-bearing waste.

The modified MVP was successfully applied to the conceptual design study of the proposed system for the assay of the steel waste discharged from the uranium enrichment plant with the activity concentration of uranium at the clearance level. The result of simulation with this code was used to draw the upper limit of background count rate due to the nat- 
ural process that makes it possible for the proposed system to confirm with the reliability that the activity of uranium in this waste is below the clearance level.

\section{Acknowledgment}

The authors are grateful to K. Sugiyama of Computer Associated Laboratory, Inc. and T. Shimada of JAEA for their extensive support in the numerical simulation. We would like to acknowledge T. Fukahori of JAEA for his help in using the photonuclear data. The present work is the result of the "Development of Active Nondestructive Assay Technique using Photofission for Uranium-Bearing Waste Discharged from Uranium Enrichment Facility" entrusted by the Ministry of Education, Culture, Sport, Science and Technology of Japan (MEXT).

\section{References}

1) Clearance Levels for Radionuclides in Solid Materials Application of Exemption Principles, IAEA-TECDOC-855, International Atomic Energy Agency (IAEA) (1996).

2) T. Gozani, Active Nondestructive Assay of Nuclear Materials - Principles and Applications NUREG/CR-0602, SAI-MLM2585 (1981).

3) A. Lyoussi, J. R. Dherbey, J. C. Edeline et al., "Low-level transuranic waste assay by photon interrogation and neutron counting," Proc. Third INMM Annual Meeting, Scottsdale, Arizona, USA, Jul. 1993, Institute of Nuclear Material Management (1993).

4) Y. Nagaya, K. Okumura, T. Mori et al., MVP/GMVP II: General Purpose Monte Carlo Codes for Neutron and Photon Transport Calculations based on Continuous Energy and Multigroup Methods, JAERI 1348, Japan Atomic Energy Research Institute (JAERI) (2005).

5) P. Vertes, D. Ridikas, "Some test calculations with IAEA photonuclear data library," J. Nucl. Sci. Technol., Supplement 2, 1049-1052 (2002).

6) M. C. White, R. C. Little, M. B. Chadwick et al., "Photonuclear pyhsics in radiation transport - II: Implementation," Nucl. Sci. Eng., 114, 174-189 (2003).

7) E. Padovani, S. D. Clarke, S. A. Pozzi, "Feasibility of prompt correlated counting from photon interrogation of concealed nuclear materials," Nucl. Instr. Meth. Phys. Res., A583, 412-420 (2007).

8) J. W. Sterbentz, J. L. Jones, W. Y. Yoon et al., "Benchmark validation comparison of measured and calculated delayed neutron detector responses for a pulsed photonuclear assesment technique," Nucl. Instr. Meth. Phys. Res., B261, 373-377 (2007).

9) Handbook on Photonuclear Data for Applications Crosssections and Spectra, final report of a co-ordinated research project 1996-1999, IAEA-TECDOC-1178 International Atomic Energy Agency (IAEA) (2000).

10) N. Kishida, T. Murata, T. Asami et al., "JENDL photonuclear data file," Proc. Int. Conf. on Nuclear Data for Science and Technology, Santa Fe, New Mexico, USA, Sept. 26 - Oct. 1, 2004 (2004).

11) V. MaLare, ENDF-102; Data Formats and Procedures for the Evaluated Nuclear Data File, ENDF-6, BNL-NCS-4494501/04-Rev., Brookhaven National Laboratory (BNL) (2001).

12) T. Mori, Y. Nagaya, K. Okumura et al., Production of MVP Neutron Cross Section Libraries Based on the Latest Evaluated Nuclear Data Files, JAERI-Data/Code 2004-011, Japan Atomic Energy Research Institute (JAERI) (2004), [in Japanese].
13) G. W. Mckinney "Tally tagging feature in MCNPX 2.7.A," Trans. ANS Winter Meeting, Reno, Nevada, USA, Nov. 9 - 13, 2008, 99[1], 633-635 (2008).

14) For instance, M. Murata, "Trend on decomissioning technology of nuclear power plants in 21st century(8);Decommissioning technologies - Technical trends of recycling contaminated metals," Nihon- Gensiryoku-Gakkai Shi (J. At. Energy Soc. Jpn.), 52[4], 219-224 (2010), [in Japanese].

15) Advisory material for the IAEA regulations for the safety transport of radioactive material: safety guide, IAEA TS-G-1.1 (ST2), International Atomic Energy Agency (IAEA) (2002).

16) R. B. Firestone, V. S. Shirley, (ed.), Table of Isotopes Eighth Edition, John Wiley \& Sons, Inc., (1996).

17) A. Blokhin, N. Buleeva, S. Nasyrova et al., "Formation and application of evaluated photoneutron data library BOFOD," Yadernye Konstanty, 3, 3 (1992).

18) A. Blokhin, Photonuclear Data Library BOFOD, Private communication (1999).

19) T. Murata, H. Matsunobu, K. Shibata, Evaluation of $(\alpha, x n)$ Reaction Data for JENDL/AN-2005, JAEA-Research 2006-052, Japan Atomic Energy Agency (JAEA) (2006).

\section{Appendix A :}

Figure 10 compares the ${ }^{235} \mathrm{U}$ and ${ }^{238} \mathrm{U}$ photofission cross sections between the IAEA library and JENDL/PD-2004. The upper limit energy of evaluation is 20 and $140 \mathrm{MeV}$ for the IAEA library and JENDL/PD-2004, respectively.

The photofission cross-section of ${ }^{238} \mathrm{U}$ in the JENDL/PD2004 is similar to that in the IAEA library in an energy range around the giant dipole resonance. But a discrepancy is observed for the ${ }^{235} \mathrm{U}$ between the two libraries near the peak of resonance.

The photonuclear data of these nuclides in the IAEA library have their origins in the BOFOD library. ${ }^{17,18)}$ These libraries have not contained the delayed neutron data due to the photofission, but the JENDL/PD-2004 provides them for the ${ }^{235} \mathrm{U}$ and ${ }^{238} \mathrm{U}$. The JENDL/PD-2004 evaluations therefore were adopted for these nuclides in the numerical simulation of present study.

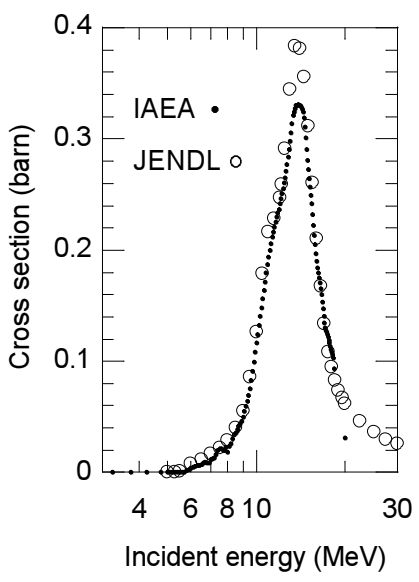

(a) ${ }^{235} \mathrm{U}$

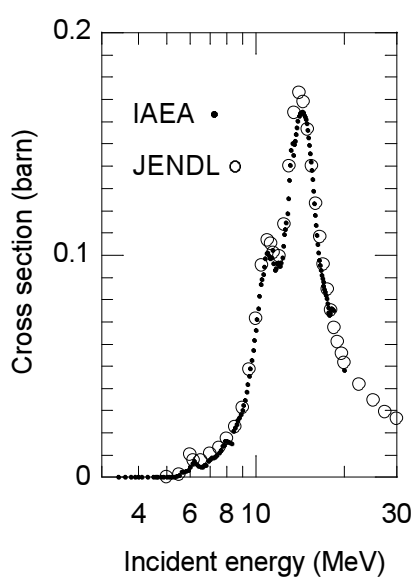

(b) ${ }^{238} \mathrm{U}$
Fig. 10 Comparison of ${ }^{235} \mathrm{U}$ and ${ }^{238} \mathrm{U}$ photofission cross sections between IAEA library and JENDL/PD-2004 
Table 4 Result of sensitivity calculation on torus model for the contaminated region (c) in the waste of $40 \mathrm{~cm}$ diameter

\begin{tabular}{ccc}
\hline Density $^{\text {a) }}$ & Counts $^{\mathrm{b})}$ & $\begin{array}{c}\text { Ratio } \\
{ }^{\mathrm{c}} \text { ) }\end{array}$ \\
\hline $1.216 \times 10^{18 \mathrm{~d})}$ & $1.0414 \times 10^{5} \pm 0.11 \%$ & $8.564 \times 10^{-14} \pm 0.11 \%$ \\
& & $(1.000)$ \\
$1.216 \times 10^{19}$ & $1.0432 \times 10^{6} \pm 0.11 \%$ & $8.579 \times 10^{-14} \pm 0.11 \%$ \\
& & $(1.002 \pm 0.14 \%)$ \\
$1.216 \times 10^{20}$ & $1.0294 \times 10^{7} \pm 0.10 \%$ & $8.465 \times 10^{-14} \pm 0.10 \%$ \\
& & $(0.988 \pm 0.14 \%)$ \\
\hline
\end{tabular}

a) Atomic number density of ${ }^{235} \mathrm{U}$ and ${ }^{238} \mathrm{U}$ summed together (atoms $/ \mathrm{cm}^{3}$ )

b) Total delayed neutron counts of 17 steps with statistical uncertainties $(1 \sigma)$ of MVP calculation

c) Ratio of the counts to the density

d) Atomic number density in original torus model

\section{Appendix B :}

A series of sensitivity calculation was carried out, where the atomic number densities of uranium isotopes in the torus region were increased by factors of 10 and 100 which cover the volume ratio between the sphere region and the corresponding torus model that were mentioned in Section IV. 3. The calculation was made for the torus model of contaminated region (c) in the waste of $40 \mathrm{~cm}$ diameter as a typical example. The delayed neutron counts were calculated with the procedure that was described in Section IV. 4. A ratio of the counts to the density was then calculated and shown in Table 4 to check that the counts linearly increased with the density.

The ratio is in agreement with that at the original density within a statistical uncertainty of $\pm 0.4 \%$ at $3 \sigma$ level when the density is increased by the factor of 10 . On the other hand, the ratio decreases by about $1 \%$ when the density is increased by the factor of 100 . From these results, a systematic uncertainty of $1 \%$ was estimated to be introduced by adopting the torus model.

\section{Appendix C :}

Table 5 shows the relative abundances and decay constants of six delayed-neutron-precursor groups for the photofissions of ${ }^{235} \mathrm{U}$ and ${ }^{238} \mathrm{U}$ in JENDL/PD-2004, which were used in the present numerical simulation.

\section{Appendix D :}

\section{Spontaneous fission}

The count rate of spontaneous fission neutrons from uranium isotopes was estimated at the required detection limit of activity concentration $0.01 \mathrm{~Bq} / \mathrm{g}$ mentioned in Section IV. 4 by the use of

(a) The activity concentration $0.01 \mathrm{~Bq} / \mathrm{g}$,

(b) The weight of waste 50,167 and $395 \mathrm{~kg}$ for the waste of 20,30 and $40 \mathrm{~cm}$ diameter, respectively, which was calculated from the volume and density of waste mentioned in Section IV. 1,

(c) Isotopic contribution to the 5\% enriched uranium activity shown in Fig. 2,

(d) Spontaneous fission branching ratio of decay of uranium isotopes, ${ }^{16)}$

(e) Spontaneous fission neutron multiplicity of about 3 and

(f) Neutron counting efficiency of the ${ }^{3} \mathrm{He}$ detectors in the present system.

The neutron counting efficiency was evaluated by a Monte Carlo transport calculation that modeled a point ${ }^{252} \mathrm{Cf}$ neutron source placed in the waste and was found to be not more than $20 \%$. Finally, the count rate was found to be at most $1.9 \times 10^{-3}, 6.6 \times 10^{-3}, 1.5 \times 10^{-2} \mathrm{cps}$ for the waste of 20,30 and $40 \mathrm{~cm}$ diameter, respectively. The spontaneous fission neutrons from the ${ }^{238} \mathrm{U}$ have a dominant contribution in this count rate because the spontaneous fission branching ratio $5.45 \times 10^{-5}$ of ${ }^{238} \mathrm{U}^{16)}$ is larger than the other uranium isotopes by a factor of more than 7,000 although the ${ }^{238} \mathrm{U}$ contribution to the $5 \%$ enriched uranium activity is $12 \%$. These count rates are far less than the background count rates at the lower detection limit shown in Table 3 by a factor of $6 \times 10^{-5}$ or less, and the contribution of spontaneous fission neutrons are neglected.

When the waste is assumed to be contaminated with the depleted uranium of above weight that is composed of ${ }^{238} \mathrm{U}$ alone, the count rates due to the spontaneous fission increase by not more than $5 \%$ because this isotope already has the dominant contribution to the counts with the $5 \%$ enriched uranium as mentioned above.

\section{2. $(\alpha, \mathbf{n})$ reaction}

It is known that the $\alpha$-particles emitted by radioactive heavy isotopes cause $(\alpha, \mathrm{n})$ reactions with 17 light isotopes, and the neutrons from this reaction are counted by the detectors during the assay of waste contaminated with the heavy isotopes in some conditions. Among them, isotopes of carbon, oxygen and fluorine are potentially included in the wastes con-

Table 5 Relative abundances $a_{i}$ and decay constants $\lambda_{i}$ of six delayed-neutron-precursor groups for the photofissions of ${ }^{235} \mathrm{U}$ and ${ }^{238} \mathrm{U}$ in JENDL/PD-2004 ${ }^{10)}$

\begin{tabular}{ccccc}
\hline Nucleus & Group $i$ & $a_{i}{ }^{\text {a) }}$ & $\lambda_{i}(/ \mathrm{s})$ & Half-life $\left.(\mathrm{s}){ }^{\mathrm{b}}\right)$ \\
\hline \multirow{3}{*}{${ }^{235} \mathrm{U}$} & 1 & 0.0549652 & 0.0131 & 52.9 \\
& 2 & 0.196396 & 0.0337 & 20.6 \\
& 3 & 0.180317 & 0.121 & 5.73 \\
& 4 & 0.387712 & 0.2952 & 2.35 \\
& 5 & 0.132426 & 0.81363 & 0.852 \\
${ }^{238} \mathrm{U}$ & 6 & 0.0481844 & 2.5721 & 0.269 \\
& 1 & 0.017841 & 0.0138 & 50.2 \\
& 3 & 0.147711 & 0.0316 & 21.9 \\
& 4 & 0.144459 & 0.1211 & 5.72 \\
& 5 & 0.209517 & 0.9073 & 0.764 \\
& 6 & 0.0940628 & 3.0368 & 0.228 \\
\hline
\end{tabular}

a) Normalized so that sum of abundances equals to unity

b) Calculated from the decay constant 
sidered in the present study. The carbon isotopes are shown in Table 2, but the other isotopes were not modeled in the numerical simulation.

In addition, the $(\alpha, \mathrm{n})$ reaction is used for a neutron source in fields of research and industry. The most widely used source is based on the $(\alpha, n)$ reaction of beryllium. Characteristics including neutron yields for the various $(\alpha, \mathrm{n})$ neutron sources are extensively reviewed in Ref. 2 ). The beryllium $(\alpha, \mathrm{n})$ sources with various $\alpha$ emitting heavy isotopes have the neutron yields between $5.4 \times 10^{-5}$ and $5.4 \times 10^{-4}(\mathrm{n} / \mathrm{s}) / \mathrm{Bq}$ of heavy isotope $\left(2 \times 10^{6}\right.$ and $2 \times 10^{7}(\mathrm{n} / \mathrm{s}) / \mathrm{Ci}$ in Ref. 2$\left.)\right)$. A typical $(\alpha, n)$ source of fluorine has the yield of $3.8 \times 10^{-5}(\mathrm{n} / \mathrm{s}) / \mathrm{Bq}$ which is similar to that of beryllium source. The source of oxygen, however, has the yield of $7.0 \times 10^{-8}(\mathrm{n} / \mathrm{s}) / \mathrm{Bq}$ which is smaller than the yields of beryllium and fluorine sources. This small yield is principally due to a small natural abundance $0.2 \%$ of ${ }^{18} \mathrm{O}$ which is the principal oxygen isotope for the $(\alpha, \mathrm{n})$ reaction, while the natural abundance is $100 \%$ for both ${ }^{9} \mathrm{Be}$ and ${ }^{19} \mathrm{~F}$.

For a carbon $(\alpha, n)$ source, the neutron yield is not given in this reference. But the yield of this source with the uranium is expected to be far less than that of the fluorine source by the following reasons :

(a) A threshold $\alpha$-particle energy of ${ }^{12} \mathrm{C}(\alpha, \mathrm{n})$ reaction is higher than the $\alpha$-particle energy of uranium isotopes which is between 4 and $5 \mathrm{MeV}$, while the threshold energy of ${ }^{13} \mathrm{C}(\alpha, \mathrm{n})$ reaction is lower than $4 \mathrm{MeV}$, and hence the ${ }^{13} \mathrm{C}$ solely contributes to the neutron emission with the uranium.

(b) The natural abundance of ${ }^{13} \mathrm{C}$ is $1.1 \%$ and less than that of ${ }^{19} \mathrm{~F}$ by a factor of 0.01 .

(c) A neutron production cross-section of ${ }^{13} \mathrm{C}(\alpha, \mathrm{n})$ reaction is of the same order of that of ${ }^{19} \mathrm{~F}(\alpha, \mathrm{n})$ one between 4 and $5 \mathrm{MeV}{ }^{19)}$

These neutron yields were used to estimate the detector counts of $(\alpha, n)$ neutrons in the waste of present study. The fluorine $(\alpha, \mathrm{n})$ reaction has dominant contribution to the $(\alpha, \mathrm{n})$ neutrons because the neutron yields of both oxygen and carbon $(\alpha, \mathrm{n})$ sources are far less than that of fluorine one as mentioned above. The count rate of detectors at the activity concentration of $0.01 \mathrm{~Bq} / \mathrm{g}$ was estimated from

(a) The neutron yields $3.8 \times 10^{-5}(\mathrm{n} / \mathrm{s}) / \mathrm{Bq}$,

(b) The activity concentration of waste $0.01 \mathrm{~Bq} / \mathrm{g}$,

(c) The weight of waste and

(d) The neutron counting efficiency $20 \%$ of the ${ }^{3} \mathrm{He}$ detectors of present system.

Finally, the count rate was found to be at most $3.8 \times 10^{-3}$, $1.2 \times 10^{-2}$ and $3.0 \times 10^{-2} \mathrm{cps}$ for the waste of 20,30 and $40 \mathrm{~cm}$ diameter, respectively. These count rates are far less than the background count rates at the lower detection limit shown in Table 3 by a factor of $1.2 \times 10^{-4}$ or less, and thus the contribution of $(\alpha, n)$ neutrons are neglected.

It should be noted that the neutron yields summarized in Ref. 2) and used in the present estimation are for the $(\alpha, n)$ sources that are mixtures of the heavy and light elements with a suitable ratio so that they efficiently work as the neutron sources. It is therefore expected that the neutron yields of $(\alpha, \mathrm{n})$ reactions in the present waste are less than those of $(\alpha, \mathrm{n})$ sources in this reference, and the neutron count rates are also less than those estimated above. 